\title{
Heavy quarkonium and quarkonium-like states at Belle and BaBar
}

\author{
A.Kuzmin ${ }^{* \dagger}$ \\ Budker Institute of Nuclear Physics, 630090, Novosibirsk, Lavrentyeva 11, Russia \\ E-mail: a.s.kuzmin@inp.nsk.su
}

We report recent results on the observation of new heavy quarkonium and quarkonium-like states from the Belle and BaBar detectors operating at the KEKB and PEP-II $e^{+} e^{-}$colliders (B-factories) at the $\Upsilon(4 S)$ energy.

XVIII International Workshop on Deep-Inelastic Scattering and Related Subjects, DIS 2010 April 19-23, 2010

Firenze, Italy

* Speaker.

$\dagger$ On behalf of the Belle collaboration 
By 2010, experiments with the Belle detector [1] at KEKB and the BaBar detector [2] at PEPII have accumulated together more than $1.5 \mathrm{ab}^{-1}$ of data. Such a data sample provides a possibility to study CP-violation in $B$-decays, charm- and $\tau$-physics. In this presentation we report results of a search for new charmonium and charmonium-like states in these experiments.

$B$-factories allow to perform a search for new states in different processes: fragmentation of light quarks $(u, d, s, c)$ in $e^{+} e^{-}$annihilation, production of new particles in $B$-meson decays, formation of $C$-even states in two-photon processes, production of $J^{P C}=1^{--}$in initial state radiation (ISR). After ten-year operation about 15 new heavy particles have been discovered including both conventional charmonia as well as exotic charmonium-like states, which cannot be explained in the frame of a simple quark model.

$\mathrm{X}(3872)$ - is the most studied charmonium-like state which does not fit the standard $q \bar{q}-$ spectroscopy. It was observed in 2003 while studying the $\pi^{+} \pi^{-} J / \psi$ mass spectrum in $B^{+} \rightarrow$ $K^{+} \pi^{+} \pi^{-} J / \psi$ decays with a sample of $152 \mathrm{M} B \bar{B}$ events [3]. In addition to a large peak corresponding to $\psi^{\prime} \rightarrow \pi^{+} \pi^{-} J / \psi$, a significant spike at $3872 \mathrm{MeV} / \mathrm{c}^{2}$ was found. A signal of $35.7 \pm 6.8$ events with a significance of $10.3 \sigma$ was observed. The mass corresponding to the peak is $M=$ $3872.4 \pm 0.6 \pm 0.5 \mathrm{MeV} / \mathrm{c}^{2}$ and an upper limit for width is $\Gamma<2.3 \mathrm{MeV}$. The existence of $X$ (3872) was confirmed by BaBar [4], CDF [5] and D0 [6]. The $\pi \pi$ invariant mass distribution is consistent with the $X(3872) \rightarrow J / \psi \rho^{0}$ intermediate state. BaBar performed a search for decays $B^{0} \rightarrow K^{-} \pi^{-} \pi^{0} J / \psi$ and $B^{-} \rightarrow K_{S} \pi^{-} \pi^{0} J / \psi$ and found no signals in the $\pi^{-} \pi^{0} J / \psi$ mass distribution [7] for both decays indicating that $X(3872)$ is an isoscalar and isospin is violated in $B \rightarrow X(3872) K$ decays.

Analyzing the data sample of $275 \mathrm{M} B \bar{B}$ events Belle found evidence for $X(3872) \rightarrow \gamma J / \psi[8]$ decays confirmed by BaBar [9]. These results established the charge-conjugation parity of $X(3872)$ as $C=+1$.

Using a larger data sample Belle examined a possible $J^{P C}$ assignment for the $X(3872)$ [10]. Angular correlations between the final state particles in $X \rightarrow \pi^{+} \pi^{-} J / \psi$ ruled out the $0^{++}$and $0^{-+}$ options. The shape of the $\pi \pi$ mass distribution favors an $S$-wave over $P$-wave as a relative orbital momentum between the final-state dipion and $J / \psi$, strongly disfavoring $1,2^{-+}$assignments. The accumulated evidence strongly favors a $1^{++}$assignment for the $X$ (3872). CDF [11] using larger data sample repeated angular correlations and $\pi \pi$ mass spectrum analysis and came to the same conclusion in the case of description of $\rho$ by simple relativistic Breit-Wigner. However if they add momentum-dependent formfactor for $\rho$ and include $\rho-\omega$-interference, both solutions with assumption of $1^{++}$and $2^{-+}$were allowed.

Some models predicts existence of an $X(3872)$-doublet. BaBar and Belle have measured $X(3872)$ production in both charged and neutral $B$-decays. No statistically significant mass difference has been found either by BaBar $\Delta M=2.7 \pm 1.6 \pm 0.4 \mathrm{MeV} / c^{2}$ [12] or by Belle $\Delta M=$ $0.18 \pm 0.89 \pm 0.26 \mathrm{MeV} / c^{2}[13]$. The ratios $\frac{\mathscr{B}\left(B^{0} \rightarrow X K^{0}\right)}{\mathscr{B}\left(B^{ \pm} \rightarrow X K^{ \pm}\right)}$measured by BaBar and Belle are $0.41 \pm$ $0.24 \pm 0.05$ and $0.82 \pm 0.22 \pm 0.05$, respectively. In 2008 Belle found a new production mode $B^{0} \rightarrow X K^{+} \pi^{-}$[13], where the $K \pi$ system is dominated by nonresonant $K \pi$ with a branching fraction product $\mathscr{B}\left(B^{0} \rightarrow X\left(K^{+} \pi^{-}\right)_{N R}\right) \mathscr{B}\left(X \rightarrow J / \psi \pi^{+} \pi^{-}\right)=8.1 \pm 2.1^{+} 1.1_{-1.4} \times 10^{-6}$ while for the $K^{*}$ contribution the upper limit was set $\mathscr{B}\left(B^{0} \rightarrow X K^{*}(892)\right) \mathscr{B}\left(X \rightarrow J / \psi \pi^{+} \pi^{-}\right)<3.4 \times 10^{-6}$.

In 2009 BaBar published the observation of the new decay mode $X(3872) \rightarrow \psi(2 S) \gamma$ with 
branching fractions product $\mathscr{B}\left(B^{ \pm} \rightarrow X K^{ \pm}\right) \times \mathscr{B}(X \rightarrow \psi(2 S) \gamma)=(9.5 \pm 2.7 \pm 0.6) \times 10^{-6}$. The observed ratio of the branching fractions $\frac{\mathscr{B}(\psi(2 S) \gamma)}{\mathscr{B}(J / \psi \gamma)}=3.4 \pm 1.4$ disfavors the $D \bar{D}^{*}$-molecule.

The proximity of the $X$ mass to $\overline{D^{*}} D$ threshold was suspicious, and eventually decays to $\overline{D^{*}} D$ were observed by BaBar [14] and Belle [15]. Both Belle and BaBar have observed $X(3872)$ signals in the $D^{* 0} \overline{D^{0}}$ final state with branching fractions about ten times higher than for $J / \psi \pi^{+} \pi^{-}$and the mass peak close to that for the latter decay.

Although X(3872) is well studied, its interpretation demands much more experimental studies. Its apparent quantum numbers, mass, and decay patterns make it an unlikely conventional charmonium candidate, and no consensus explanation has been found yet.

In 2005 analysing the spectrum of recoil mass to $J / \psi$ Belle [16] found both the peaks corresponding to the well-known $\eta_{c}, \chi_{c 0}, \eta_{c}(2 S)$ states and a peak with mass of about $3940 \mathrm{MeV} / \mathrm{c}^{2}$. It was shown that it does not decay to $J / \psi \omega$ and is not the same particle as $\mathrm{Y}(3940)$ discovered by Belle [17] in $B^{-} \rightarrow J / \psi \omega K^{-}$and confirmed by BaBar [18]. Belle measurement [19] with the reconstruction of the $D \bar{D}^{*}$ system gave $M=3942_{-6}^{+7} \pm 6 \mathrm{MeV} / c^{2}$ and width $\Gamma=37_{-15}^{+26} \pm 8 \mathrm{MeV}$. Analysing data on $e^{+} e^{-} \rightarrow J / \psi D^{*} \bar{D}^{*}$ Belle also observed a new state decaying to $D^{*} \bar{D}^{*}$ with mass $M=4156_{-20}^{+25} \pm 15 \mathrm{MeV} / c^{2}$ and width $\Gamma=139_{-61}^{+111} \pm 21 \mathrm{MeV}$.

Rich results on $C$-even states are provided by a study of two-photon collisions. In 2006 Belle observed a peak in the $D \bar{D}$ system with the angular distribution consistent with a spin-2 hypothesis and mass and width $M=3929 \pm 5 \pm 2 \mathrm{MeV} / c^{2} \Gamma=29 \pm 10 \pm 2 \mathrm{MeV}$ [20]. This measurement has been confirmed by BaBar which mass and width are consistent with Belle: $M=3926.7 \pm 2.7 \pm$ 1.1 MeV $/ c^{2} \Gamma=21.3 \pm 6.8 \pm 3.6 \mathrm{MeV}[21]$. This state can be interpreted as the $\chi_{c 2}(2 P)$-state.

Belle searched in two-photon collisions for the Y(4143) particle observed by CDF [22] and set upper limits of $\Gamma_{\gamma \gamma} \mathscr{B}(Y \rightarrow \phi J / \psi)<36(5.3) \mathrm{eV}$ for $\mathrm{J}^{\mathrm{P}}=0^{+}\left(2^{+}\right)$[23]. However, evidence of another peak with a significance of $3.2 \sigma$ with mass and width $M=4350.6_{-5.1}^{+4.6} \pm 0.7 \mathrm{MeV} / c^{2} \Gamma=$ $13_{-9}^{+18} \pm 4 \mathrm{MeV}$ was found. The interpretation of this peak is unclear and more studies are necessary. Analysis of another final state in $\gamma \gamma \rightarrow \omega J / \psi$ performed by Belle showed the presence of the peak with mass and width of $M=3914 \pm 3 \pm 2 \mathrm{MeV} / c^{2} \Gamma=23 \pm 10_{-8}^{+2} \mathrm{MeV}$ [24]. Its interpretation is also unclear.

Five new states with $1^{--}$quantum numbers have been observed in ISR processes [25]-[31]. The masses, widths and decay modes of these states are listed in Table 1. All these states cannot be interpreted as conventional charmonia because all $1^{--}$in this energy range have been already discovered and assigned and neither of these new states have decay modes with open charm.

The $Z^{+}$(4430) was observed by Belle [32] in 2007 in $B \rightarrow \psi(2 S) \pi^{-} K$ decay in the $\psi(2 S) \pi^{-}$ mass spectrum after removing events corresponding to the $K^{*}(892)$ and $K^{*}(1430)$ states. Mass and width $M=4433 \pm 4 \pm 2 \mathrm{MeV} / c^{2} ; \quad \Gamma=45_{-13-33}^{+18+30} \mathrm{MeV}$ and the branching fraction product $\mathscr{B}\left(\overline{B^{0}} \rightarrow Z^{+} K\right) \mathscr{B}\left(Z^{+} \rightarrow \psi(2 S) \pi^{+}\right)=(4.1 \pm 1.0 \pm 1.4) \times 10^{-5}$ were measured. BaBar [33] did not confirm $Z^{+}$and set an upper limit on this particle production $\mathscr{B}\left(\overline{B^{0}} \rightarrow Z^{+} K\right) \mathscr{B}\left(Z^{+} \rightarrow\right.$ $\left.\psi(2 S) \pi^{+}\right)<(3.1) \times 10^{-5}$. However, this upper limit is just $1.7 \sigma$ part from the Belle value. Belle performed Dalitz plot analysis [34] of $B \rightarrow \psi(2 S) \pi^{-} K$ including the contributions of various $K \pi$ states $\left(\kappa, K^{*}(892), K^{*}(1410), K_{0}^{*}(1430), K_{2}^{*}(1430), K^{*}(1680)\right)$ and confirmed Z(4430) existence with mass and width shown in Table 1 and the branching fraction product $\mathscr{B}\left(\bar{B}^{0} \rightarrow Z^{+} K\right) \mathscr{B}\left(Z^{+} \rightarrow\right.$ $\left.\psi(2 S) \pi^{+}\right)=\left(3.2_{-0.9-1.6}^{+1.8+5.3}\right) \times 10^{-5}$. Fits with and without $Z^{+}$have probabilities $36 \%$ and $0.1 \%$, respectively. 
A.Kuzmin

\begin{tabular}{|c|c|c|c|c|c|}
\hline State & $\overline{\mathrm{M}(\mathrm{MeV})}$ & $\bar{\Gamma} \Gamma(\mathrm{MeV})$ & $\overline{J^{P C}}$ & Process (mode) & Experiment \\
\hline \multicolumn{6}{|c|}{ B-decays } \\
\hline$\eta_{c}(2 S)$ & $3637 \pm 4$ & $14 \pm 7$ & $0^{-+}$ & $K\left(K_{S}^{0} K^{-} \pi^{+}\right)$ & Belle(2002), BaBar, CLEO \\
\hline$X(3872)$ & $3871.52 \pm 0.20$ & $1.3 \pm 0.6$ & $1^{++} / 2^{-+}$ & $\begin{array}{l}K\left(\pi^{+} \pi^{-} J / \psi\right) \\
K\left(D^{* 0} \overline{D^{0}}\right), \ldots\end{array}$ & Belle(2003), BaBar,CDF,D0 \\
\hline$Y(3940)$ & $3915.7 \pm 4.2$ & $41 \pm 12$ & $0 / 2^{?+}$ & $K(\omega J / \psi)$ & Belle(2004), BaBar \\
\hline$\overline{Z_{1}(4050)^{+}}$ & $4051_{-43}^{+24}$ & $82_{-55}^{+51}$ & $?$ & $K\left(\pi^{+} \chi_{c 1}(1 P)\right)$ & Belle (2008) \\
\hline$Z_{2}(4250)^{+}$ & $4248_{-45}^{+185}$ & $177_{-72}^{+321}$ & $?$ & $K\left(\pi^{+} \chi_{c 1}(1 P)\right)$ & Belle (2008) \\
\hline$Z(4430)^{+}$ & $4443_{-18}^{+24}$ & $107_{-71}^{+113}$ & $?$ & $K\left(\pi^{+} \psi(2 S)\right)$ & Belle (2007) \\
\hline \multicolumn{6}{|c|}{ Double charmonium } \\
\hline$X(3940)$ & $3942_{-8}^{+9}$ & $37_{-17}^{+27}$ & $?++$ & $J / \psi\left(D \bar{D}^{*}\right)$ & Belle (2007) \\
\hline$X(4160)$ & $4156_{-25}^{+29}$ & $139_{-65}^{+113}$ & $? ?^{?+}$ & $J / \psi\left(D^{*} D^{*}\right)$ & Belle (2007) \\
\hline \multicolumn{6}{|c|}{ ISR } \\
\hline$Y(4008)$ & $4008_{-49}^{+121}$ & $226 \pm 97$ & $1^{--}$ & $\left(\pi^{+} \pi^{-} J / \psi\right)$ & Belle (2007) \\
\hline$Y(4260)$ & $4263 \pm 5$ & $108 \pm 14$ & $1^{--}$ & $\left(\pi^{+} \pi^{-} J / \psi\right)$ & BaBar (2005), Belle, CLEO \\
\hline$Y(4360)$ & $4353 \pm 11$ & $96 \pm 42$ & $1^{--}$ & $\left(\pi^{+} \pi^{-} \psi(2 S)\right)$ & BaBar (2007), Belle \\
\hline$X(4630)$ & $4634_{-11}^{+9}$ & $92_{-32}^{+41}$ & $1^{--}$ & $\left(\Lambda_{c} \Lambda_{c}\right)$ & Belle (2007) \\
\hline$Y(4660)$ & $4664 \pm 12$ & $48 \pm 15$ & $1^{--}$ & $\left(\pi^{+} \pi^{-} \psi(2 S)\right)$ & Belle (2007) \\
\hline \multicolumn{6}{|c|}{ Two-photons } \\
\hline$\chi_{c 2}(2 P)$ & $3927.2 \pm 2.6$ & $24.1 \pm 6.1$ & $2^{++}$ & $(D \bar{D})$ & Belle(2005), BaBar \\
\hline $\mathrm{X}(3915)$ & $3914 \pm 4$ & $23_{-13}^{+10}$ & $0,2^{++}$ & $(\omega J / \psi)$ & Belle(2009) \\
\hline$X(4350)$ & $4350.6_{-5.1}^{+4.6}$ & $13.3_{-10.0}^{+18.4}$ & $0,2^{++}$ & $(\phi J / \psi)$ & Belle(2009) \\
\hline \multicolumn{6}{|c|}{ Energy scan and $\Upsilon$ transitions } \\
\hline$\eta_{b}(1 S)$ & $9390.7 \pm 2.9$ & $?$ & $0^{-+}$ & $\gamma+(\ldots)$ & BaBar(2008), CLEO \\
\hline$Y_{b}$ & $10889.6 \pm 2.3$ & $54.7_{-7.6}^{+8.9}$ & $1^{--}$ & $\pi^{+} \pi^{-} \Upsilon(n S)$ & Belle (2008) \\
\hline
\end{tabular}

Table 1: Heavy quarkonia discovered at $B$-factories

Another two charged $Z$-states with masses shown in Table 1 were observed in the $\overline{B^{0}} \rightarrow$ $K^{-} \pi^{+} \chi_{c 1}$ Dalitz plot analysis performed by Belle [35].

The observed charmonium-like charged $Z$ states are particularly interesting. If these states were mesons, they would necessarily have a minimal 4-quark substructure of $c \bar{c} u \bar{d}$ and therefore be exotic.

The list of all heavy-quarkonium states discovered at Belle and BaBar is listed in Table 1. Now the list contains more than 15 states. The data analysis is in progress and more states are expected to be discovered. Super B-factories will give more data and more experimental information for understanding the nature of the new states.

\section{References}

[1] A. Abashian et al. (Belle Collaboration), Nucl. Instr. and Meth. A 479 (2002) 117.

[2] B. Aubert et al. [BABAR Collaboration], Nucl. Instrum. Meth. A 479 (2002) 1. 
[3] S. K. Choi et al. (Belle Collaboration), Phys. Rev. Lett. 91 (2003) 262001.

[4] B. Aubert et al. (BaBar Collaboration), Phys. Rev. D 77 (2008) 111101.

[5] D. E. Acosta et al. (CDF II Collaboration), Phys. Rev. Lett. 93 (2004) 072001.

[6] V. M. Abazov et al. (D0 Collaboration), Phys. Rev. Lett. 93 (2004) 162002.

[7] B. Aubert et al. (BaBar Collaboration), Phys. Rev. D 71 (2005) 031501.

[8] K. Abe et al. (Belle Collaboration), [arXiv:hep-ex/0505037].

[9] B. Aubert et al. (BaBar Collaboration), Phys. Rev. Lett. 102 (2009) 132001.

[10] K. Abe et al. (Belle Collaboration), [arXiv:hep-ex/0505038].

[11] A. Abulencia et al. (CDF Collaboration), Phys. Rev. Lett. 96 (2006) 102002.

[12] B. Aubert et al. (BaBar Collaboration), Phys. Rev. D 77 (2008) 111101.

[13] I. Adachi et al. (Belle Collaboration), arXiv:0809.1224 [hep-ex].

[14] B. Aubert et al. (BaBar Collaboration), Phys. Rev. D 77 (2008) 011102.

[15] T. Aushev et al. (Belle Collaboration), Phys. Rev. D 81, 031103 (2010).

[16] K. Abe et al. (Belle Collaboration), Phys. Rev. Lett. 98 (2007) 082001.

[17] K. Abe et al. (Belle Collaboration), Phys. Rev. Lett. 94 (2005) 182002.

[18] B. Aubert et al. (BaBar Collaboration), Phys. Rev. Lett. 101 (2008) 082001.

[19] P. Pakhlov et al. (Belle Collaboration), Phys. Rev. Lett. 100 (2008) 202001.

[20] S. Uehara et al. (Belle Collaboration), Phys. Rev. Lett. 96 (2006) 082003.

[21] B. Aubert (BaBar Collaboration), arXiv:1002.0281 [hep-ex].

[22] T. Aaltonen et al. (CDF Collaboration), Phys. Rev. Lett. 102 (2009) 242002.

[23] C. P. Shen et al. (Belle Collaboration), Phys. Rev. Lett. 104 (2010) 112004.

[24] S. Uehara et al. (Belle Collaboration), Phys. Rev. Lett. 104 (2010) 092001.

[25] B. Aubert et al. (BaBar Collaboration), Phys. Rev. Lett. 95 (2005) 142001.

[26] B. Aubert et al. (BaBar Collaboration), arXiv:0808.1543v2 [hep-ex].

[27] C. Z. Yuan et al. (Belle Collaboration), Phys. Rev. Lett. 99 (2007) 182004.

[28] B. Aubert et al. (BaBar Collaboration), Phys. Rev. Lett. 98 (2007) 212001.

[29] X. L. Wang et al. (Belle Collaboration), Phys. Rev. Lett. 99 (2007) 142002.

[30] G. Pakhlova et al. (Belle Collaboration), Phys. Rev. Lett. 101 (2008) 172001.

[31] G. Pakhlova et al. (Belle Collaboration), Phys. Rev. D 80 (2009) 091101.

[32] S. K. Choi et al. (Belle Collaboration), Phys. Rev. Lett. 100 (2008) 142001.

[33] B. Aubert et al. (BaBar Collaboration), Phys. Rev. Lett. 102 (2009) 012001.

[34] R. Mizuk et al. (Belle Collaboration), Phys. Rev. D 80 (2009) 031104.

[35] R. Mizuk et al. (Belle Collaboration), Phys. Rev. D 78 (2008) 072004. 